\title{
Pulmonary coinfection of Mycobacterium tuberculosis and Tropheryma whipplei: a case report
}

\author{
Binghua Zhu' ${ }^{1}$, Jing Tang ${ }^{2}$, Rong Fang ${ }^{1}$, Xuejie Fei ${ }^{1}$, Qing Wang ${ }^{1}$, Wenqing Wang ${ }^{1}$, Xueqin Wu ${ }^{3}$, Chao Liu ${ }^{3 *}$ (D) and
} Qian Wang ${ }^{1 *}$

\begin{abstract}
Background: We diagnosed a clinical case of pulmonary infection involving Mycobacterium tuberculosis and Tropheryma whipplei in a patient with acute respiratory distress syndrome. The diagnosis was assisted by metagenomic next-generation sequencing of bronchoalveolar lavage fluid.

Case presentation: A 44-year-old Han Chinese inmate was transferred to the emergency department because of dry cough, chest tightness, and shortness of breath. The patient's body temperature rose to $39.3^{\circ} \mathrm{C}$ following empirical cephalosporin treatment for 1 week. The blood CD4+/CD8+ ratio was 0.7 , suggesting immunodeficiency. Routine microbiological tests were performed, and tuberculosis interferon gamma release assays were positive. Mycobacterium tuberculosis polymerase chain reaction was also positive. Chest computed tomography scan revealed miliary nodules and ground-glass opacifications, which were in accordance with tuberculosis. To fully examine the etiology, we performed routine laboratory tests and metagenomic sequencing, the results of which indicated the presence of Mycobacterium tuberculosis and Tropheryma whipplei. We administered anti-tuberculosis regimen in combination with trimethoprim/sulfamethoxazole. The patient recovered, with chest computed tomography scan showing absorption of lesions.
\end{abstract}

Conclusions: Compared with traditional diagnostic methods such as culture and serology, metagenomic next-generation sequencing has the advantage of detecting a wide array of microorganisms in a single test and therefore can be used for clinical diagnosis of rare pathogens and microbial coinfections. It is particularly useful for immunocompromised patients as they are more prone to infection by opportunistic microorganisms.

Keywords: Mycobacterium tuberculosis, Tropheryma whipplei, Pulmonary infection, Metagenomic next-generation sequencing

\section{Background}

Here we review a case of community-acquired pneumonia caused by Mycobacterium tuberculosis (MTB) and

\footnotetext{
*Correspondence: zenith.fenix@gmail.com; docqianwang@126.com 1 Shuguang Hospital Affiliated to Shanghai University of Traditional Chinese Medicine, Shanghai 200021, China

${ }^{3}$ Hangzhou Matridx Biotechnology Co., Ltd, Bd 5, 208 Zhenzhong Road, Hangzhou 311113, Zhejiang, China

Full list of author information is available at the end of the article
}

Tropheryma whipplei (TW). MTB is a species of pathogenic bacteria in the family Mycobacteriaceae and the causative agent of tuberculosis [1]. TW is a Gram-positive Actinobacteria and the causative agent of Whipple's disease [2]. TW is commonly found in the environment and can be carried by $1.5-7 \%$ of healthy individuals without causing symptoms [3]. MTB may occur concomitantly with other infections such as human immunodeficiency virus (HIV) [4]. However, clinical reports of MTB and TW coinfection are scarce. original author(s) and the source, provide a link to the Creative Commons licence, and indicate if changes were made. The images or other third party material in this article are included in the article's Creative Commons licence, unless indicated otherwise in a credit line to the material. If material is not included in the article's Creative Commons licence and your intended use is not permitted by statutory regulation or exceeds the permitted use, you will need to obtain permission directly from the copyright holder. To view a copy of this licence, visit http://creativecommons.org/licenses/by/4.0/. The Creative Commons Public Domain Dedication waiver (http://creativeco mmons.org/publicdomain/zero/1.0/) applies to the data made available in this article, unless otherwise stated in a credit line to the data. 


\section{Case presentation}

A 44-year-old male patient (Han Chinese ethnicity) experienced dry cough, chest tightness, and shortness of breath for 2 weeks and was transferred to the emergency department at Shuguang Hospital. The patient has been previously diagnosed with type 2 diabetes and hepatitis $\mathrm{C}$ and had a history of intravenous drug use. Upon admission, we conducted a physical examination that showed a body temperature of $39.3{ }^{\circ} \mathrm{C}$, pulse of 117 beats per minute, respiratory rate of $22-30$ breaths per minute, and blood pressure of $120 / 84 \mathrm{mmHg}$. The patient had normal development and body shape, and was conscious but showed signs of malnutrition. Skin was normal without yellow dye, erythema, rash, or pigmentation. Lymph glands were not enlarged. Chest auscultation indicated rough breath sounds and moist rales in both lungs. Furthermore, bronchoscopy and chest computed tomography $(\mathrm{CT})$ scan revealed miliary nodules and ground-glass opacifications in both lungs (Fig. 1a, b).

Next, we carried out routine laboratory tests, and the results were as follows: $\mathrm{SpO}_{2} 90 \%$ (breath via facial mask, FiO2 50\%), $\mathrm{PaO}_{2} 52 \mathrm{mmHg}, \mathrm{PaCO}_{2} 26 \mathrm{mmHg}, \mathrm{pH} 7.5$, Base Excess (BE) $0.6 \mathrm{mmol} / \mathrm{L}$, Lactate concentration (Lac) $2.1 \mathrm{mmol} / \mathrm{L}$, Oxygenation Index (OI) $101 \mathrm{mmHg}$, white blood cell count $(\mathrm{WBC}) 7.3 \times 10^{9} / \mathrm{L}$, Neutrophil $(\mathrm{N})$ 89\%, C-reactive protein (CRP) $111.7 \mathrm{mg} / \mathrm{L}$, procalcitonin (PCT) $0.67 \mathrm{ng} / \mathrm{ml}$, total bilirubin (TBiL) $31.8 \mu \mathrm{mol} / \mathrm{L}$, alanine aminotransferase (ALT) $59 \mathrm{U} / \mathrm{L}$, aspartate aminotransferase (AST) $135 \mathrm{U} / \mathrm{L}$, albumin (Alb) $24 \mathrm{~g} / \mathrm{L}$, and CD4+/CD8+ 0.7. The microbiological culture of sputum reported Gram-positive and Gram-negative cocci and Candida albicans. Acid-fast stain, Mycoplasma pneumoniae tests were negative. Bacterial and fungal culture of bronchoalveolar lavage fluid (BALF) were negative. Galactomannan test (GM test) using BALF was negative. Blood culture was negative. Serum $\beta$-D-Glucan test (G test) was negative. Tuberculosis immunoglobulin $M$ (IgM) and immunoglobulin G (IgG) tests were negative. However, the interferon alpha release assays including T-SPOT.TB and QuantiFERON-TB Gold (QFT-G) were positive. As a result, we performed metagenomic nextgeneration sequencing (mNGS) tests on BALF (DNA sequencing), which reported one read of Mycobacterium tuberculosis and 43 reads of Tropheryma whipplei. To further confirm the presence of MTB, we carried out MTB fluorescent polymerase chain reaction (PCR) test, which was positive for MTB with no detection of rifampicin and isoniazid antibiotic resistance (Table 1).

Combining clinical manifestation and above-mentioned microbiological data, we diagnosed the patient with community-acquired pneumonia with acute respiratory distress syndrome (ARDS) that was caused by coinfection of MTB and TW. We then performed endotracheal intubation and mechanical ventilation and

Table 1. Results of MTB fluorescent PCR

\begin{tabular}{lll}
\hline Test & Molecular target & Result \\
\hline MTB & rpoB gene & Positive \\
Rifampicin resistance & rpoB AA 507-512 mutation & Negative \\
& rpoB AA 512-520 mutation & Negative \\
& rpoB AA 520-528 mutation & Negative \\
& rpoB AA 528-533 mutation & Negative \\
& katG (315 G>C) & GG \\
Isoniazid resistance & katG $(-15 \mathrm{C}>\mathrm{T})$ & $\mathrm{CC}$ \\
\hline
\end{tabular}

MTB: Mycobacterium Tuberculosis, AA: amino acid
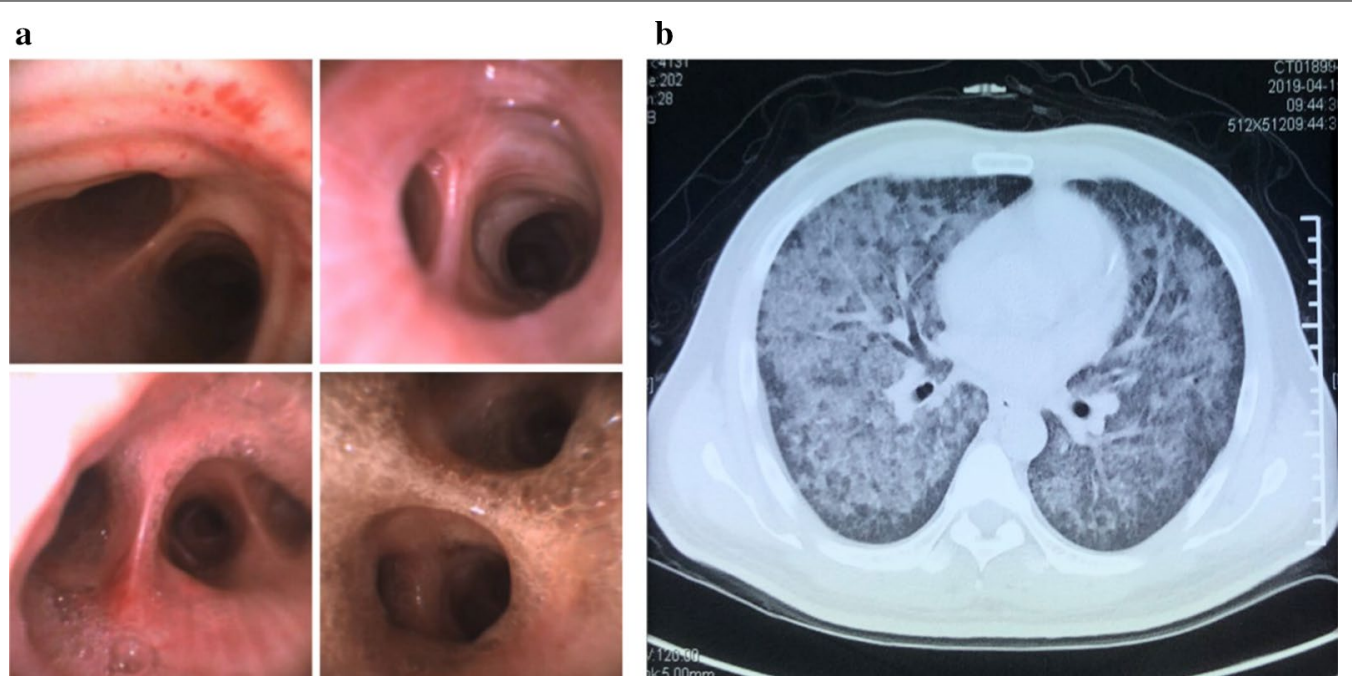

Fig. 1 Bronchoscopy and chest computed tomography image. a. Images taken during bronchoscopy showing miliary nodules. b. Chest computed tomography image showing ground-glass opacifications in both lungs 
administered trimethoprim/sulfamethoxazole (TMP/ SMX) $1.44 \mathrm{~g}$ three times per day orally, isoniazid $0.3 \mathrm{~g}$ once per day orally, rifapentine $0.6 \mathrm{~g}$ twice per week orally, amikacin 0.6 once per day intravenous guttae (ivgtt), and ethambutol $0.75 \mathrm{~g}$ once per day orally.

After 14 days of treatment, the patient's body temperature returned to normal with improvement of blood oxygen saturation. Chest $\mathrm{CT}$ scan displayed absorption of ground-glass opacifications (Fig. 2a). Ventilator was removed, and the patient was given high-flow nasal cannula. At 21 days posttreatment, chest CT scan showed significant absorption of miliary nodules (Fig. 2b).

\section{Discussion and conclusions}

Tuberculosis may lead to severe sepsis, which can be fatal in patients with immunodeficiency, including patients with HIV, cancer, and immunological disorders [5]. TW could cause both acute and chronic infections and can colonize healthy individuals. On the other hand, TW is known to cause acute pneumonia, which was previously reported to be associated with aspiration pneumonia as well as acute respiratory distress syndrome (ARDS) [6]. TW infection may initially lead to fever and idiopathic or migratory arthritis and progress to cause steatorrhea, weight loss, hepatosplenomegaly, and ascites. Notably, approximately $30 \%$ of TW infection affects the lung, resulting in respiratory symptoms including dry cough, chest pain, shortness of breath, and pleural adhesion. There are at least 11 clinical reports involving TW and interstitial lung disease [7]. In a study using mNGS and BALF samples collected from 210 intensive care unit (ICU) patients with pneumonia, six (3\%) contained sequencing reads specifically aligned to TW [8]. In addition, TW is commonly found in HIV patients, who have poses a higher risk of pulmonary infection [9]. Considering that pulmonary alveoli contain macrophages, which TW mainly targets, they may serve as primary sites of TW colonization or infection.

Diagnosis of TW is usually difficult owing to the paucity of microbiological tests. The antibody titer is low in serum, making a serology test ineffective at diagnosing TW infection [10]. Periodic acid-Schiff (PAS) stain has been a traditional and primary diagnostic method for TW. However, the presence of other pathogens including Pneumocystis jirovecii and Mycobacterium avium may produce false-positive results [11]. Moreover, TW is auxotrophic and takes $\sim 18$ hours for one proliferation cycle, making bacterial culture impractical. However, with the development of nucleic acid tests, PCR-based and sequencing-based molecular assays have proven to be effective for TW detection and, hence, have become the main diagnostic choice [12].

Guidelines for treating TW infection are currently lacking. Literature suggests a variety of antimicrobial regimen, including penicillin 12,000 units/day [13], tetracycline $600 \mathrm{mg} /$ day [14], TMP/SMX 160/800 mg/ day [15], TMP/SMX 160/800 mg/day with ceftriaxone $2 \mathrm{~g} /$ day or meropenem $3 \mathrm{~g} /$ day [16], and doxycycline $200 \mathrm{mg} /$ day with hydroxychloroquine $600 \mathrm{mg} /$ day [15]. In our case, the patient has type 2 diabetes and has been serving a sentence in prison. According to one study, the incidence rate of MTB infection in prison $(657 / 100,000)$ is 3.75 -fold higher than in the general population [17]. The malnutrition state, positive for blood T-SPOT.TB and QFT-G tests, as well as miliary nodules seen on chest $\mathrm{CT}$ were all consistent


Fig. 2 Chest computed tomography images after treatment. a. Chest computed tomography after 14 days of treatment. b. Chest computed tomography after 21 days of treatment 
with tuberculosis. The diagnosis was further corroborated by mNGS and PCR. The low read number may be due to difficulties in nucleic acid extraction, as the cell walls of MTB are rich in lipid and hard to break by either chemical or physical means. In addition to miliary nodules, ground-glass opacifications were also evident on chest CT that indicate infection of additional pathogens. The ratio of $C D 4+/ C D 8+$ was less than 1 , suggesting an immunodeficient state of the patient. We initially did not administer anti-TB treatment but were able to observe some absorption of exudation around miliary nodules after use of antibiotics. By adding antiTB therapy, most pulmonary lesions were absorbed within 3 weeks (Fig. 2b). Moreover, we ruled out pulmonary alveolar hemorrhage and proteinosis because of the following evidence: (1) blood was not present in BALF; (2) the patient has been diagnosed with hepatitis $C$, which was classified as Child-Pugh class A with normal international normalized ratio (INR); (3) BALF was clear, and no precipitates were seen after centrifugation; and (4) PAS stain was negative. As a result, combining these clinical observations with presence of MTB and TW sequencing reads by mNGS, we concluded that the patient had a pulmonary coinfection of MTB and TW.

In summary, TW is less commonly detected in the general population than in immunocompromised individuals. Opportunistic infection and coinfection of TW is possible. Pulmonary TW infection is usually indistinguishable from other bacterial infections. As a result, nucleic acid tests such as mNGS are especially valuable for identification and differential diagnosis of TW infection. We also confirmed in our case that oral administration of TMP/SMX was effective in treating pulmonary TW infection.

\begin{abstract}
Abbreviations
mNGS: Metagenomic next-generation sequencing; MTB: Mycobacterium tuberculosis; TW: Tropheryma whipplei; ARDS: Acute respiratory distress syndrome; TMP/SMX:Trimethoprim/sulfamethoxazole; BALF: Bronchoalveolar lavage fluid.

\section{Acknowledgements}

Not applicable.

\section{Authors' contributions}

BZ and QW were the primary physicians that provided clinical diagnosis and treatment of the patient. JT, RF, XF, QW, and WW analyzed and interpreted the patient data. XW and CL provided metagenomic sequencing and analyzed results. BZ and CL wrote the manuscript. All authors read and approved the final manuscript.
\end{abstract}

\section{Funding}

No funding was received for this case report.

\section{Declarations}

Ethics approval and consent to participate

We received approval by the board of Ethics at Shuguang Hospital Affiliated to Shanghai University of Traditional Chinese Medicine.

\section{Consent for publication}

Written informed consent was obtained from the patient for publication of this case report and any accompanying images. A copy of the written consent is available for review by the Editor-in-Chief of this journal.

\section{Competing interests}

The authors declare no conflicts of interests.

\section{Author details}

${ }^{1}$ Shuguang Hospital Affiliated to Shanghai University of Traditional Chinese Medicine, Shanghai 200021, China. ${ }^{2}$ Shanghai Guanghua Hosptial of Integrated Traditional Chinese and Western Medicine, Shanghai 200052, China. ${ }_{3}^{3}$ Hangzhou Matridx Biotechnology Co., Ltd, Bd 5, 208 Zhenzhong Road, Hangzhou 311113, Zhejiang, China.

Received: 17 May 2020 Accepted: 10 May 2021

Published online: 09 July 2021

\section{References}

1. Gordon SV, Parish T. Microbe profile: Mycobacterium tuberculosis: humanity's deadly microbial foe. Microbiology. 2018;164(4):437-9.

2. La Scola B, et al. Description of Tropheryma whipplei gen. nov., sp. Nov., the Whipple's disease bacillus. Int J Syst Evol Microbiol. 2001;51(Pt 4):1471-9.

3. Puechal X. Whipple's disease. Ann Rheum Dis. 2013;72(6):797-803.

4. Deffur A, Mulder NJ, Wilkinson RJ. Co-infection with Mycobacterium tuberculosis and human immunodeficiency virus: an overview and motivation for systems approaches. Pathog Dis. 2013;69(2):101-13.

5. Devaraj NK, et al. The effects of probiotic supplementation on the incidence of diarrhea in cancer patients receiving radiation therapy: a systematic review with meta-analysis and trial sequential analysis of randomized controlled trials. Nutrients. 2019;11(12):2886.

6. Lagier JC, et al. Tropheryma whipplei DNA in bronchoalveolar lavage samples: a case control study. Clin Microbiol Infect. 2016;22(10):875-9.

7. Urbanski $G$, et al. Whipple disease revealed by lung involvement: a case report and literature review. Chest. 2012;141(6):1595-8.

8. Bousbia S, et al. Tropheryma whipplei in patients with pneumonia. Emerg Infect Dis. 2010;16(2):258-63.

9. Lozupone C, et al. Widespread colonization of the lung by Tropheryma whipplei in HIV infection. Am J Respir Crit Care Med. 2013;187(10):1110-7.

10. Desnues B, Al Moussawi K, Fenollar F. New insights into Whipple's disease and Tropheryma whipplei infections. Microbes Infect. 2010;12(14-15):1102-10.

11. AIDS with Mycobacterium avium-intracellulare lesions resembling those of Whipple's disease. N Engl J Med, 1983. 309(21): 1323-5.

12. Fenollar F, et al. Use of genome selected repeated sequences increases the sensitivity of PCR detection of Tropheryma whipplei. J Clin Microbiol. 2004;42(1):401-3.

13. Keinath $\mathrm{RD}$, et al. Antibiotic treatment and relapse in Whipple's disease. Long-term follow-up of 88 patients. Gastroenterology. 1985;88(6):1867-73.

14. Bai JC, et al. Short-term antibiotic treatment in Whipple's disease. J Clin Gastroenterol. 1991;13(3):303-7.

15. Lagier JC, et al. Treatment of classic Whipple's disease: from in vitro results to clinical outcome. J Antimicrob Chemother. 2014;69(1):219-27.

16. Feurle GE, Junga NS, Marth T. Efficacy of ceftriaxone or meropenem as initial therapies in Whipple's disease. Gastroenterology. 2010;138(2):478-86.

17. Sanchez A, et al. Extensive Mycobacterium tuberculosis circulation in a highly endemic prison and the need for urgent environmental interventions. Epidemiol Infect. 2012;140(10):1853-61.

\section{Publisher's Note}

Springer Nature remains neutral with regard to jurisdictional claims in published maps and institutional affiliations. 